\title{
Blood Pressure and Heart Rate Changes in Different Body Figures during Submaximal Exercise
}

\author{
Rujia Yi ${ }^{1}$, Jianan Zhang ${ }^{2}$, Xiaolu Ye ${ }^{3}, Y_{\text {ong }} \mathrm{Xie}^{4}$,Fengyuan Zou ${ }^{5}$
}

\begin{abstract}
In order to analyze blood pressure and heart rate changes during submaximal exercise in different body figures, 36 female college students underwent treadmill exercise with heart rate being monitored and blood pressure measured before, immediately after and 10 minutes after exercise. Correlation analysis between body mass index, bust and waist variation, torso volume index and relevant parameters demonstrated results that, body mass index is superior to other indexes as a predictor of rest HR and BP responses; BWV was a better predictor of peak HR and average HR during exercise; TVI better predicts HR recovery after exercise. When stratified by torso volume index, people with normal weight tend to perform better than other groups.
\end{abstract}

Keywords: Body figure; TVI; female; blood pressure; heart rate

\section{Introduction}

As the method and technology of body figure analysis develops, the body figure index was increasingly applied to scientific inquiries. The influence of body figure on the

\footnotetext{
${ }^{1} \mathrm{R} . \mathrm{Yi}$

Zhejiang Sci-Tech University, Hangzhou, China

e-mail:only018@hotmail.com

2 J. Zhang

Zhejiang Sci-Tech University, Hangzhou, China

e-mail: ganny.zhang@hotmail.com

${ }^{3} \mathrm{X}$. Ye

Zhejiang Sci-Tech University, Hangzhou, China

e-mail: yxlmyyx@163.com

4 Y.Xie

Zhejiang Sci-Tech University, Hangzhou, China

e-mail:valiantxy@126.com

5 F. Zou $(\bowtie)$

Zhejiang provincial research center of clothing engineering technology, Zhejiang Sci-Tech University,

Hangzhou, China

e-mail: zfy166@hotmail.com
} 
indexes of body function is admittedly significant, but was often confined to body mass index (BMI) and waist circumference (WC). Research by Gul Ar Navi Khan et al (2013) examined various blood pressure parameters' changes during exercise in young medical students in different BMI groups [1]; the Coventry University studied the associations between BMI, WC and a body shape index (ABSI) with resting blood pressure [2] However, grouping by TVI is unprecedented. Thus, the aim of this study was to examine the association between BMI, bust and waist variance (BWV), TVI and blood pressure, heart rate, and to consider the role of BMI, BWV and TVI during submaximal exercise in order to determine which one provides a better predictor of the indexes of body function.

\section{Method}

\subsection{Subjects}

36 healthy female college students aged 20 to 25 years, with a normal cardiovascular system and hadn't taken any cardioactive drug recently [3]; not in the menstrual cycle.

\subsection{Equipment}

After scanned by American [TC] $]^{2}$ 3-D body measuring instrument, all subjects exercised on a treadmill(Phoenix JDB-1060D). The treadmill was placed in a constant temperature and humidity laboratory, with temperature set at $20^{\circ} \mathrm{C} \pm 3^{\circ} \mathrm{C}$ and relative humidity at $65 \% \pm 5 \%$. Heart rates were constantly monitored by Polar RS400 training computer, while blood pressures were obtained from the left arm before and after exercise by MEC-1000 portable multi-parameter monitor.

\subsection{Body figure measurement}

Body shape indexes were measured before treadmill exercise so as to classify the subjects. All students stood inside a darkroom dressed in light color underclothes with their feet and hands on markers. The apparatus captured thousands of data points, extract measurement data automatically and present them on the computer. We extracted data of body volume, back neck height, inseam height, bust and waist for calculation. 


\subsection{Treadmill protocol}

The rest heart rate and blood pressure were obtained during standing so that data wouldn't be affected by different postures [4]. The protocol used the Bruce treadmill test (a standardized procedure used to evaluate the cardiovascular fitness of athletes) [5] for reference but altered according to preliminary experiment. The speed was set at $4 \mathrm{~km} / \mathrm{h}$ at the beginning, with an elevation of $1 \mathrm{~km} / \mathrm{h}$ at the end of each minute of exercise. Exercise was terminated after proceeding for five minutes where target heart rate was achieved, defined as $85 \%$ of their maximal heart rate. Gait was maintained by a metronome at a setting of 120 [4]. Subjects were instructed not to hold on to the rail in order to avoid dissipation of work force and to keep an even mode. Blood pressure were measured at the minute and 10 minutes after exercise terminated.

\subsection{Data analysis}

Variables are presented as means \pm SD. A probability $(\mathrm{P})$ value of $<0.05$ was considered significant.

Body form data: The body form parameter used in this study was height, weight, bust, waist, torso volume (TV) and torso length (TL), which were derived from the 3-D body measuring instrument. $\mathrm{BMI}=$ weight $/$ height $^{2}, \mathrm{BWV}=$ bust-waist, $\mathrm{TVI}=\mathrm{TV} / \mathrm{TL}^{2}$.

BMI, BWV and TVI were used as quantitative indicators to assess one's weight status. TVI could be construed as ratio of mean transection area to torso length that eliminate the influence of limbs and head form [6].

Subjects were classified into three groups according to TVI as follows: Group A (5.09-6.70), underweight, 12 subjects; group B (6.70-8.20), normal range, 17 subjects; Group C (8.20-9.70), overweight, 7 subjects. The obese group was excluded because the subjects were too small to include in statistical analysis.

Heart rate parameters: Heart rate (HR) parameters include rest HR, peak HR, average HR and value for HR recovery. Define HR recovery indexes as follows: (1) decrease in HR from peak HR to $T$ minute after exercise: $\Delta \mathrm{HR}_{\mathrm{T}}=$ Peak HR-HR $\mathrm{T}$; (2) Slope of HR recovery after $\mathrm{T}$ minutes' recovery [7]: $\mathrm{HR}_{\mathrm{T}}=$ peak $\mathrm{HR}+\beta \mathrm{T}$; (3) Relative decrease in $\mathrm{HR}$ at time $\mathrm{T}$ after exercise:

$\triangle \mathrm{HRT} \%=\frac{\text { Peak HR }-\mathrm{HRT}}{\text { Peak HR }- \text { Rest HR }} \times 100 \%$.

Blood pressure parameters: Blood pressure parameters comprise of systolic BP (SBP) and diastolic $\mathrm{BP}(\mathrm{DBP})$ at time $\mathrm{T}$ recorded as $\mathrm{SBP}_{\mathrm{T}}$ and $\mathrm{DBP}_{\mathrm{T}}[8]$. 
3 Results and discussion

\subsection{Characteristics at base line}

The base-line characteristics are summarized in Table 1.

Table 1 Baseline Characteristics of Study Subjects

\begin{tabular}{ll}
\hline Variable & $\mathrm{n}=12$ \\
\hline Height $(\mathrm{cm})$ & $160.2 \pm 0.1$ \\
Weight $(\mathrm{kg})$ & $49.7 \pm 5.8$ \\
BMI & $19.4 \pm 2.1$ \\
BWV $(\mathrm{cm})$ & $14.5 \pm 4.0$ \\
TVI & $7.1 \pm 1.1$ \\
Rest HR(beats/min $)$ & $87.8 \pm 6.3$ \\
SBP $(\mathrm{mm} \mathrm{Hg})$ & $111.2 \pm 9.4$ \\
DBP $(\mathrm{mm} \mathrm{Hg})$ & $70.8 \pm 8.1$ \\
\hline
\end{tabular}

\subsection{Somatotype}

The correlation between HR, BP parameters and somatotype indexes were evaluated as shown in Table 2. The correlation between TVI and HR recovery was prominently significant $(\mathrm{P}<0.01)$. BMI was correlated significantly with rest $\mathrm{HR}$ and $\mathrm{BP}(\mathrm{P}<0.01)$, and BWV was correlated significantly with peak HR and average $\mathrm{HR}(\mathrm{P}<0.01)$.

Table 2 Correlations between Body Figure Indexes and BP, HR

\begin{tabular}{llll}
\hline & TVI & BMI & BWV \\
\hline Rest HR & 0.296 & $0.516^{* *}$ & 0.261 \\
Peak HR & 0.241 & 0.244 & $0.467^{* *}$ \\
Average HR & 0.189 & 0.154 & $0.474^{* *}$ \\
$\Delta \mathrm{HR}_{1} \%$ & $0.562^{* * *}$ & 0.112 & -0.105 \\
$\mathrm{SBP}$ & 0.241 & $0.465^{* *}$ & 0.090 \\
$\mathrm{DBP}^{*}$ & 0.053 & 0.129 & 0.083 \\
$\mathrm{SBP}_{5}$ & $0.349^{*}$ & $0.549^{* *}$ & 0.075 \\
$\mathrm{DBP}_{5}$ & 0.095 & 0.132 & -0.244 \\
\hline
\end{tabular}




\subsection{Body mass index}

The correlation between BMI and rest HR, BP was significant according to correlation analysis. The correlation between BMI and BP has been proved according to many studies that BMI was a predictor of risk of elevated BP, whereas the mechanism of BMI's influence on rest HR remained to be determined.

\subsection{Bust and waist variance}

BWV was significantly related to peak HR and average HR during exercise. According to previous studies, BWV or bust-waist ratio (BWR) was not significantly related to body function indexes such as BP or blood sugar. The conclusion was also verified in this study.

\subsection{Torso volume index}

Heart rate response: Regression analysis identified that TVI was prominently significant in quadratic correlation to $\Delta \mathrm{HR}_{1} \quad(\mathrm{P}<0.01)$, with regression equation of $\mathrm{Y}=1.70+0.57 \mathrm{X}-0.04 \mathrm{X}^{2}$.

The correlations between HR recovery parameters, rest $\mathrm{HR}$ and peak HR were contained in Table 3. $\Delta \mathrm{HR}_{2}$ was excluded since it was not significantly related to other indexes. As can be seen in Table 2, there was significant relation between peak HR and

Table 3 Correlations Among HR Recovery Variables

\begin{tabular}{llllll}
\hline & Peak HR & $\Delta \mathrm{HR}_{1}$ & $\Delta \mathrm{HR}_{1} \%$ & $\beta$ & $\Delta \mathrm{HR}_{2}$ \\
\hline Rest HR & $0.40^{*}$ & 1.26 & -0.22 & $0.35^{*}$ & -0.17 \\
Peak HR & & 0.24 & -0.19 & -0.17 & 0.28 \\
$\Delta \mathrm{HR}_{1}$ & & $0.87^{* *}$ & $-0.90^{* *}$ & $0.78^{* * *}$ \\
$\Delta \mathrm{HR}_{1} \%$ & & & $-0.78^{* *}$ & $0.66^{* *}$ \\
$\beta$ & & & & $-0.77^{* * *}$ \\
\hline
\end{tabular}

rest HR $(\mathrm{P}<0.017)$, while slope of HR recovery was significantly related to rest $\mathrm{HR}$ $(\mathrm{P}<0.05)$.

According to t-test, Group A and Group B performed a significant difference $(\mathrm{P}<0.05)$.

Heart rate curve was fitted as shown in Figure 1 with regard to mean HR of each group respectively. The curves exhibit similar tendencies, while Group C displayed higher HR value compared to other groups. Similarly, HR of Group C at base-line was higher; the value of decrease in HR after exercise was smaller. As for HR recovery period, Group B 
has higher value for HR recovery.

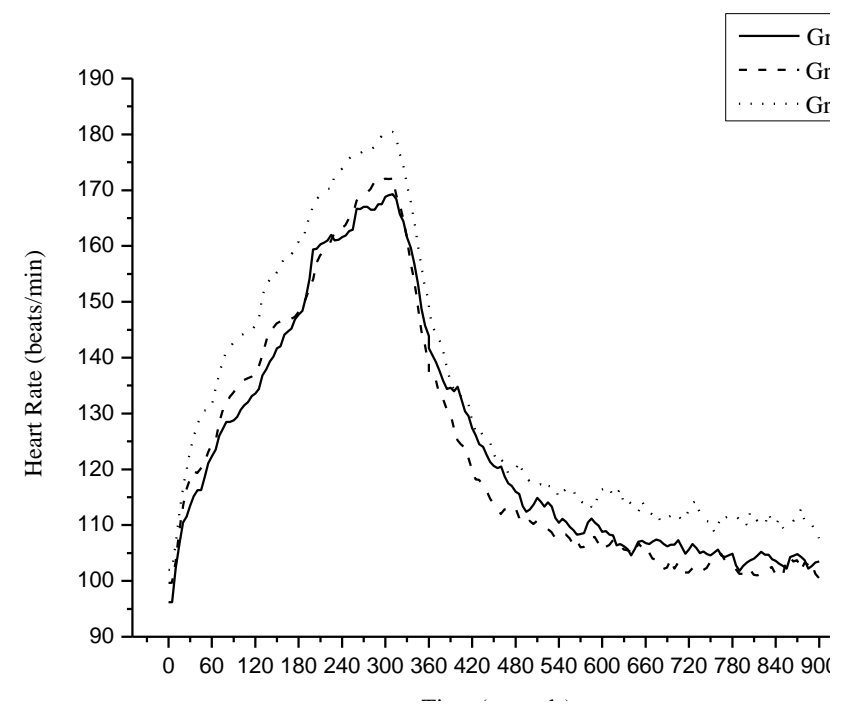

Fig. 1 Stratified HR Curve during Exercise

Analysis on HR curve demonstrated that subjects tend to have the highest increase in HR during the first minute of exercise with comparison to other groups. Because treadmill protocol was set in expectation to achieve a state of submaximal exercise, the increase in HR slowed down till it achieved $85 \%$ of maximal HR. Peak HR of Group A differ significantly from Group C, but not from Group B, while relative decrease in HR after exercise of Group B differ significantly from other groups $(\mathrm{P}<0.05)$. Group $\mathrm{C}$ has higher mean peak HR than Group A and Group B (180.7, 173.5 and 173.3 beats/min respectively)

Heart rate response reflected functional status of autonomic nervous system, according to study. The increase in HR that accompanies exercise is due to a reduction in vagal tone, the decrease in HR immediately after exercise is a function of vagal reactivation [9]. Heart rate response during exercise can be prognostic indicator of mortality.

Obesity performed negative impact on vagal activity and balance between sympathetic nerve and vagus nerve [10]. Thus, under relatively high work load, the overweight people have lower vagal activity but higher sympathetic activity, which explains for the lower HR recovery value than other groups. Since the underweight group has lower HR recovery rate than normal group, we assume that vagal activity and the balance as mentioned were also affected negatively. 
Blood pressure response: Stratified blood pressures were shown in Table 4. Group C has higher rest SBP $(116.7 \pm 11.6 \mathrm{~mm} \mathrm{Hg})$ than Group A $(109.6 \pm 8.7 \mathrm{~mm} \mathrm{Hg})$ and Group B (110.1 $\pm 8.6 \mathrm{~mm} \mathrm{Hg})$, where DBP was not differed prominently. After ten minutes' recovery, BP reverts to rest BP, even lower than rest BP. SBP1, DBP1 and SBP10, DBP10 were significantly correlated to SBP0 and $\mathrm{DBP} 0(\mathrm{P}=0.001, \mathrm{P}=0.000 ; \mathrm{P}=0.008, \mathrm{P}=0.000)$.

Table 4 Baseline and Exercise Blood Pressure

\begin{tabular}{llllllc}
\hline & \multicolumn{2}{c}{ Before exercise } & \multicolumn{2}{c}{ Immediately after exercise } & \multicolumn{2}{c}{10 minutes after exercise } \\
\cline { 2 - 6 } & SBP & DBP & SBP & DBP & SBP & DBP \\
\hline Group A & $109.6 \pm 8.7$ & $71.1 \pm 8.0$ & $125.4 \pm 14.8$ & $76.3 \pm 6.5$ & $109.3 \pm 13.0$ & $72.4 \pm 7.1$ \\
Group B & $110.1 \pm 8.6$ & $69.2 \pm 8.5$ & $128.5 \pm 11.5$ & $77.9 \pm 6.6$ & $107.2 \pm 7.8$ & $70.0 \pm 6.8$ \\
Group C & $116.7 \pm 11.6$ & $74.1 \pm 7.5$ & $138.3 \pm 16.5$ & $77.1 \pm 6.1$ & $112.6 \pm 15.0$ & $72.4 \pm 5.9$ \\
\hline
\end{tabular}

\section{Conclusions}

BMI was superior to other indexes as a predictor of rest HR and BP responses; BWV was a better predictor of peak HR and average HR during exercise; TVI better predicts HR recovery after exercise.

Subjects with various TVI underwent a submaximal exercise tend to differ in heart rate and blood pressure responses. Specifically, females with TVI of 6.70-8.20 have higher value of HR recovery, those who with TVI over 8.20 have worse $H R$ and BP performances than other groups. Though considering physical condition, exercise frequency and genetic factor, the conclusion might be somewhat restrictive.

Acknowledgment. This work is supported by Professor Feng-yuan Zou, the corresponding author of this paper.

\section{References}

${ }^{1}$ Gul Ar Navi Khan, et al. Effect of Exercise on Various Blood Pressure Parameters in different Groups of Body Mass Index among Young Medical Students. Indian Journal of Public Health Research \& Development, 2013 4:93-97.

${ }_{2}$ Michael J. Duncan, Jorge Mota, Susana Vale, et al. Associations between body mass index, waist 
circumference and body shape index with resting blood pressure in Portuguese adolescents. Annals of Human Biology, 2013, 40(2):163-167.

${ }^{3}$ Xue-fang Yu. Evaluation on normal heart rate range of healthy adults. Chinese Journal of Practical Medicine, 2008, 35(15):57-58.

${ }^{4}$ Donald A. Riopel, Ashby B. Taylor, Arno R. Hohn. Blood Pressure, Heart Rate, Pressure-Rate Product

and Electrocardiographic Changes in Healthy Children During Treadmill Exercise. The American Journal

of Cardiology, 1979, 44: 697-704.

${ }^{5}$ Bruce RA, Kusuml F, Hosmer D. Maximal oxygen intake and monographic assessment of functional aerobic impairment in cardiovascular disease. American Heart Journal, 1973, 85:546-562.

${ }^{6}$ Yunchu Yang, Minzhi Chen, Fengyuan Zou. Female body shape parameters analysis based on 3-D scan data. Journal of Textile Research.2009, 8(30):117-122.

${ }^{7}$ Morshedi-Meibodi A, Larson MG, levy D et al. Heart rate recovery after treadmill exercise testing and risk of cardiovascular disease events. The American Journal of Cardiology, 2002, 90(8):848-852.

${ }^{8}$ Gang Wang, Zhihong Zhang, Xiaoqun Liu, et al. Analysis of changes and influences on blood pressure and heart rate before and after maximal exercise of undergraduates. Modern Preventive

Medicine,2012,39(20):5315-5319.

${ }^{9}$ Christopher R. Cole, M.D., Eugene H. Blackstone, M.D., Fredric J. Pashkow, M.D., et al. Heart-Rate Recovery Immediately after Exercise as a Predictor of Mortality. The New England Journal of Medicine, 1999, 341:1351-1357.

${ }^{10}$ Haiqiu Chen. The study on obese adolescents' heart autonomic nerve function of different exercise load. Beijing Sport University,2012. 